\title{
Thermodynamic Properties of 3-Methyl-4H-Pyrazole and its Corresponding Radicals
}

\author{
JAT RAKESH KUMAR* and SHARMA SUNIL
}

Institute of Pharmaceutical Science, Shri JJT University Vidyanagari, Churu Jhunjhunu Road Chudela, District-Jhunjhunu, Rajasthan-333001, India

rakeshkumarrrjpt@gmail.com

Received 10 February 2016 / Accepted 26 February 2016

\begin{abstract}
Thermodynamic calculations have been performed on 3-methyl- $4 H$-pyrazole to obtain insight of its thermochemical properties like its bond energies, enthalpy of formation, entropy and heat capacities. By using different computational methods some of the properties have been studied. Heat of formation was calculated by using MOPAC and Gaussians program. All calculation has been done to study these properties at a range of temperature (298 K-1500 K). Gas phase $\Delta \mathrm{H}_{\mathrm{f}}$ values found in this study are the following (in $\mathrm{kcal} / \mathrm{mol}$ ): for parent molecule $\left(\mathrm{yccdnndc}-\mathrm{CH}_{3}\right.$ ) is 54.47 , for yccdnndc-ch2j 91.13, for yccjdnnc- $\mathrm{CH}_{3} 112.84$ and 89.7 for ycjednnc- $\mathrm{CH}_{3}$.
\end{abstract}

Keywords: Internal rotor analysis, 3-Methyl- $4 H$-pyrazole, Guassian, MOPAC

\section{Introduction}

Diazole derivatives studied from the long time for their use in propellant and explosive use ${ }^{1}$. They have advantage of producing $\mathrm{N}_{2}$ as a product and reducing $\mathrm{CO}_{2}$ and carbonic acid erosion in gun barrels ${ }^{1-9}$. Several other derivatives of diazole has their use in the pesticides and synthesis of various synthetic drug molecule.3-methyl- $4 \mathrm{H}$-pyrazole has been chosen for the study due to wide spread use of this kind of molecules and there is no thermochemical study done on this particular molecule ${ }^{2}$. In this study, we tried to explore a number of thermochemical properties of 3-methyl-4H-pyrazole.

Properties like enthalpies $\left(\Delta \mathrm{H}_{\mathrm{f}}\right)$, entropies and heat capacities $(\mathrm{Cp}(\mathrm{T}))$ were determined for parent molecule $\left(\mathrm{yC}_{3} \mathrm{H}_{6} \mathrm{~N}_{2}\right)$ and its radicals, which represent loss of one hydrogen from the parent structure from the possible site of detachment. Internal rotor analysis also carried out in order to study its potential barrier and its contribution on the heat capacities and entropy. The input matrix for SMCPS (Statistical Mechanics for Heat Capacity and Entropy) got from the frequencies data obtained from Gaussians method using B3LYP/6-31G (d,p). The Guassian view of parent and its radical gives an insight of the structures.

\section{Experimental}

The choice of method was based on the property we are looking for and the level of accuracy desired. We determined the heat of formation, entropy and heat capacity of 3-methyl- $4 \mathrm{H}$ - 
pyrazole and its radicals. The first part of the study was to determine the heat of formation by using MOPAC7 PM3 and GaussiansB3LYP/6-31G(d,p). Initial calculations were done using MOPAC by incorporating $Z$-matrix in the program. Although MOPAC is not so precise method for the calculation but it helped us to make the work reactions and more importantly it provides us template to run the Gaussians program.

\section{Parent $\left(\mathrm{C}_{4} \mathrm{H}_{6} \mathrm{~N}_{2}\right)$ radical 1}
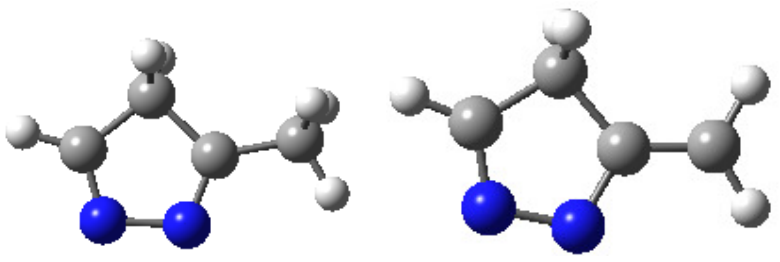

Radical 2, Radical 3
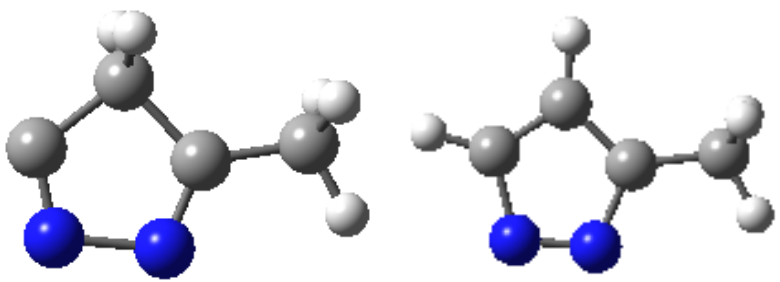

The Z-matrix has been optimized before running MOPAC and Gaussian calculations. Structural parameters for these species were optimized for structure at the B3LYP/6-31G (d,p) level of theory. The MOPAC and Gaussian methods were performed by initial geometry optimization and frequency calculations with HF theory. By studying the structure of 3methyl-4H-pyrazole, we designed optimized Z-matrix by putting value for the bond length, bond angle, dihedral angle and the connections. The following parameters were obtained using this calculation.

\section{Enthalpies of formation}

First enthalpies of formation were calculated by using MOPAC and Gaussians calculations. Then work reactions have been designed in such a way that can provide the more accurate heat of formation. Work reactions involved reactant and product with the same bond type. This use of same kind of bond types in all the molecules involved reduced the chances of error to the great extent as reactant and product has same bonding environment. Heat of formation of all reactant and products were determined.

$$
\Delta \mathrm{H}_{\mathrm{rxn}}=\Sigma \Delta \mathrm{H}_{\text {products }}-\Sigma \Delta \mathrm{H}_{\text {reactants }}
$$

By using above equation, experimental $\Delta \mathrm{H}_{\mathrm{rxn}}$ has been determined. Putting the literature value available and $\Delta \mathrm{H}_{\mathrm{rxn}}$, enthalpy of formation for the parent molecule has been determined (Table 1). Same methodology were used for calculating respective heat of formation. Work reactions were designed one for parent molecule and three for the respective radicals.

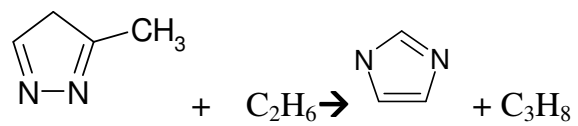

Or we can write this equation as in string format yccdnndc- $\mathrm{CH}_{3}+\mathrm{Ch} 3 \mathrm{Ch} 3 \rightarrow$ ycncdendc $+\mathrm{CH}_{3} \mathrm{CH}_{2} \mathrm{dCH}_{3}$ 
Work reaction for radicals

2) Radical 1:

$$
\text { yccdnndc- } \mathrm{CH}_{2} \mathrm{j}+\mathrm{CH}_{3} \mathrm{CH}_{2} \mathrm{dCH}_{3} \rightarrow \text { yccdnndc- }-\mathrm{CH}_{3}+\mathrm{CH}_{2} \mathrm{dCHCH}
$$

3) Radical 2:

$$
\text { yccjdnndc- } \mathrm{CH}_{3}+\mathrm{CH}_{2} \mathrm{dCH}_{2} \rightarrow \text { yccdnndc- } \mathrm{CH}_{3}+\mathrm{CH}_{2} \mathrm{dCHj}
$$

4) Radical 3:

$$
\text { ycjcdnndc- } \mathrm{CH}_{3}+\mathrm{CH}_{2} \mathrm{dCHCH}_{3} \Rightarrow \text { yccdnndc-ch3 }+\mathrm{CH}_{2} \mathrm{dCHCH}_{2} \mathrm{j}
$$

\section{Entropies and heat capacities}

Entropies and heat capacities were determined by incorporating the geometry, symmetry, frequencies and moments of inertia of the B3LYP/6-31G(d,p) optimized structures in the statistical mechanics SMCPS program. Frequencies used in SMCPS got from the Gaussian derived frequency values.

\section{Results and Discussion}

MOPAC and Gaussian B3LYP calculation were performed to obtain the heat of formation. For MOPAC program, $Z$-matrix was designed. Parallel calculation were done

\begin{tabular}{|c|c|c|}
\hline Reactions & $\begin{array}{c}\Delta \mathrm{H}_{\mathrm{frxn}}, \mathrm{kcal} / \mathrm{mol} \\
\text { MOPAC }\end{array}$ & $\begin{array}{l}\Delta \mathrm{H}_{\mathrm{frxn}}, \mathrm{kcal} / \\
\mathrm{mol} \mathrm{B} 3 \mathrm{LYP}\end{array}$ \\
\hline$y c c d n n d c-\operatorname{ch} 3+\operatorname{ch} 3 \operatorname{ch} 3 \Rightarrow y \operatorname{cncdcnde}+\operatorname{ch} 3 \operatorname{ch} 2 d \operatorname{ch} 2$ & -24.46 & -26.16 \\
\hline yccdnndc-ch $2 j+\operatorname{ch} 2 d \operatorname{chch} 3 \Rightarrow y c c d n n d c-\operatorname{ch} 3+\operatorname{ch} 2 d \operatorname{chch} 2 j$ & -5.77 & -1.43 \\
\hline y ccjdnndc-ch $3+\operatorname{ch} 2 d \operatorname{ch} 2 \Rightarrow y c c d n n d c-c h 3+\operatorname{ch} 2 d \operatorname{chj}$ & -15.25 & 0.09 \\
\hline$y$ cjednndc-ch $3+\operatorname{ch} 2 d \operatorname{chch} 3 \Rightarrow y c c d n n d c-c h 3+\operatorname{ch} 2 d \operatorname{chch} 2 j$ & -1.88 & 0.0 \\
\hline
\end{tabular}
by applying command thermo and aigout to obtain various physical and thermal properties value and obtained the input program for Guassian (Table 2). Same Guassian B3LYP carried out for parent molecule including the radicals and all potential reactants employed in work reaction.

Table 1. $\Delta \mathrm{H}_{\mathrm{rxn}}$ reaction obtained after putting value of heat of formation from the MOPAC and Gaussian

Work reactions were designed to get the $\Delta \mathrm{H}_{\mathrm{rxn}}$ by inputting values obtained from MOPAC and Guassian. Separate calculation were made for both, but using the same work reaction. Care was taken to use the reactant with same bonding environment as of the molecule undergoes study. 
Table 2. Calculated heat of formation by using $\Delta \mathrm{H}_{\mathrm{rxn}}$ value and the resulted heat of formation

\begin{tabular}{ccc}
\hline Species & $\Delta \mathrm{H}_{\mathrm{f}}, \mathrm{kcal} \mathrm{mol}^{-1}$ & MOPACB3LYP \\
\hline yccdnndc-ch3 & 52.77 & 54.47 \\
yccdnndc-ch2j & 94.57 & 91.13 \\
y ccjdnndc-ch3 & 126.48 & 112.84 \\
y cjcdnndc-ch3 & 90.68 & 89.7 \\
\hline
\end{tabular}

\section{Moment of inertia}

Moments of inertia from Gaussian and MOPAC of parent molecule and its radical have been recorded (Table 3). As data from Gaussian is much accurate and reliable than MOPAC, hence Gaussian moment of inertia has been used in SMCPS calculation.

Table 3. Moment of inertia of pyrazole and its radicals

\begin{tabular}{ccccc}
\hline & $\begin{array}{c}\text { Parent Guassian } \\
(\mathrm{GHz})\end{array}$ & $\begin{array}{c}\text { Radical 1 } \\
\text { Guassian }(\mathrm{GHz})\end{array}$ & $\begin{array}{c}\text { Radical 2 } \\
\text { Gaussian }(\mathrm{GHz})\end{array}$ & $\begin{array}{c}\text { Radical 3 } \\
\text { Gaussian }(\mathrm{GHz})\end{array}$ \\
\hline A & 8.7292107 & 8.7272233 & 8.6539830 & 8.6675295 \\
B & 3.4464156 & 3.5887036 & 3.5831795 & 3.7872009 \\
C & 2.5483751 & 2.6312454 & 2.6174387 & 2.6790641 \\
\hline
\end{tabular}

For parent molecule (yccdnndc-ch3), Radical 1 (yccdnndc-ch2j) Radical 2 (y ccjdnndc-ch3)

\section{SMCPS analysis}

Heat capacities and entropy was calculated using SMCPS method. The contribution of internal rotor in the molecule was determined by using VIBIR. By obtaining the data of internal rotor contribution final correction has been done in the heat capacities and entropy. We have done SMCPS calculations with rotor and without rotor. The results are presented in Table 4 and Figure 1.

Table 4. Heat capacity comparison of MOPAC and smcps for parent molecule (yccdnndc-ch3)

\begin{tabular}{ccc}
\hline Temperature, $\mathrm{K}$ & $\mathrm{C}_{\mathrm{p}}, \mathrm{cal} / \mathrm{k} / \mathrm{mol}, \mathrm{MOPAC}$ & $\mathrm{C}_{\mathrm{p}}, \mathrm{cal} / \mathrm{k} / \mathrm{mol} \mathrm{SMCPs}$ (1rotor) \\
\hline 298 & 21.7 & 19.8 \\
398 & 28.1 & 26.3 \\
498 & 33.8 & 31.8 \\
598 & 38.4 & 36.4 \\
698 & 42.2 & 40.2 \\
798 & 45.4 & 43.4 \\
898 & 48.1 & 46.1 \\
998 & 50.3 & 48.3 \\
1098 & 52.3 & 50.3 \\
1198 & 53.9 & 51.9 \\
1298 & 55.4 & 53.4 \\
1398 & 56.6 & 54.6 \\
1498 & 57.7 & 55.7 \\
\hline
\end{tabular}




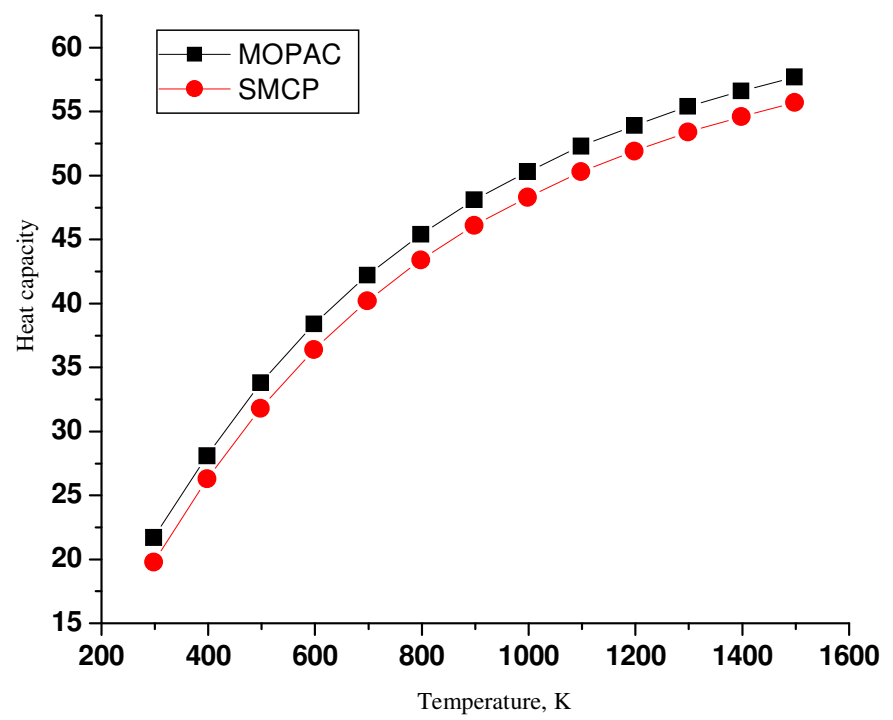

Figure 1. Heat capacities vs. temperature

The difference between heat capacity calculated from both the method was very low, but, the difference is much higher in the entropy calculated from both method as can be seen in Figure 2.

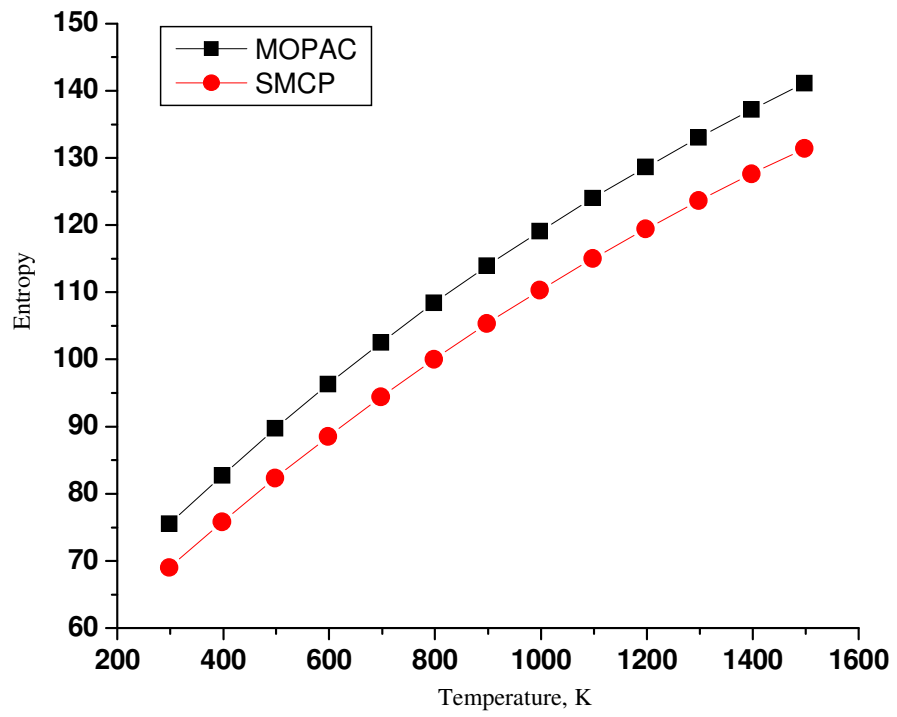

Figure 2. Comparison of entropy calculated with MOPAC and SMCP.

To correct the theomochemical properties for the variation in internal rotor, we added the internal rotor calculation for the individual molecules and corrected the entropy and enthalpy data (Table 5).

Revised heat capacities, entropy of all molecules including the contribution of rotor obtained from VIBIR. As we seen above there is difference between heat capacity and entropy obtained for molecules with and without rotor using SMCPS program. 
Table 5. Final heat capacities and entropy obtained for the parent molecule and its radicals

\begin{tabular}{ccccccccc}
\hline Temp, K & $\begin{array}{c}\text { Parent } \mathrm{C}_{\mathrm{p}}, \\
\text { cal/k/mol }\end{array}$ & $\begin{array}{c}\mathrm{S} \\
(\text { Parent })\end{array}$ & $\begin{array}{c}(\mathrm{R} 1) \\
\mathrm{C}_{\mathrm{p}}\end{array}$ & $\begin{array}{c}\mathrm{S} \\
(\mathrm{R} 1)\end{array}$ & $\begin{array}{c}(\mathrm{R} 2) \\
\mathrm{C}_{\mathrm{p}}\end{array}$ & $\mathrm{S}(\mathrm{R} 2)$ & $\begin{array}{c}(\mathrm{R} 3) \\
\mathrm{C}_{\mathrm{p}}\end{array}$ & $\mathrm{S}$ (R3) \\
\hline 300 & 20.8 & 74.5 & 20.1 & 73.9 & 21.1 & 76.2 & 20.1 & 72.8 \\
400 & 27.3 & 75.8 & 26.6 & 75.3 & 26.7 & 77.4 & 26.6 & 73.9 \\
500 & 32.8 & 82.3 & 31.8 & 81.6 & 31.5 & 83.7 & 31.8 & 80.2 \\
600 & 37.4 & 88.5 & 36.0 & 87.6 & 35.5 & 89.6 & 36.0 & 86.2 \\
700 & 41.0 & 94.4 & 39.1 & 93.3 & 38.6 & 95.2 & 39.1 & 91.8 \\
800 & 44.3 & 100.0 & 42 & 98.6 & 41.6 & 100.4 & 42 & 97.1 \\
900 & 47.0 & 105.3 & 44.2 & 103.5 & 43.8 & 105.3 & 44.2 & 102.1 \\
1000 & 49.2 & 110.3 & 46.1 & 108.2 & 45.8 & 110.0 & 46.2 & 106.8 \\
1100 & 51.2 & 115.0 & 47.8 & 112.6 & 47.5 & 114.3 & 47.8 & 111.2 \\
1200 & 52.8 & 119.4 & 49.2 & 116.7 & 48.9 & 118.4 & 49.2 & 115.3 \\
1300 & 54.3 & 123.6 & 50.4 & 120.6 & 50.2 & 122.3 & 50.4 & 119.2 \\
1400 & 55.5 & 127.6 & 51.5 & 124.3 & 51.3 & 126.0 & 51.5 & 122.9 \\
1500 & 56.6 & 131.4 & 52.2 & 127.9 & 52.0 & 129.5 & 52.2 & 126.4 \\
\hline
\end{tabular}

\section{Conclusion}

Isodesmic work reaction used to reduce the error in the calculation of heat of formation. Specific work reaction has been designed by using reactants having the same bonding environment as of target molecule. This made it possible to get the more accurate value of heat of formation of the molecule.

This study also shows that there is significant difference in the thermochemical properties value by using semi empirical method (PM3). So, it is evident that as the molecule goes complex the difference in the MOPAC and Gaussian goes increase. Hence, the error is high in MOPAC analyses when we worked on complex molecule. But, overall for initial calculations MOPAC is a useful tool, whereas Gaussian along with SMCPS and VIBIR are used for the advance level calculations.

\section{References}

1 Gabriel da Silva, Eric E Moore and Joseph W Bozzelli, J Phys Chem A, 2006, 110, 13979-13988; DOI:10.1021/jp065150w

2 GellertMezei and Raphael G Rabtis, Inorganica Chimica Acta, 2004, 357(11), 32793288; DOI:10.1016/j.ica.2004.03.016

3 Manuvesh Sangwan and Lev N Krasnoperov, J Phys Chem A, 2013, 117(14), 29162923; DOI:10.1021/jp4000889

4 Manuvesh Sangwan, Evgeni N Chesnokov and Lev N Krasnoperov, J Phys Chem A, 2012, 116(34), 8661- 8670; DOI:10.1021/jp305070c

$5 \quad$ Manuvesh Sangwan and Lev N Krasnoperov, J Phys Chem A, 2012, 116(48), 11817 11822; DOI:10.1021/jp308885j

6 Manuvesh Sangwan, Evgeni N. Chesnokov and Lev N.Krasnoperov, J Phys Chem A, 2012, 116(24), 6282-6294; DOI:10.1021/jp211805v

7 Manuvesh Sangwan, Chao Yan, Evgeni N Chesnokov and Lev N Krasnoperov, $J$ Phys Chem A, 2015, 119(28), 7847-7857; DOI:10.1021/acs.jpca.5b01276

8 Kamilah Hylton, Manuvesh Sangwan and Somenath Mitra, Analytica Chimica Acta, 2009, 653(1), 116-120; DOI:10.1016/j.aca.2009.08.042

9 Lei Zhu, Manuvesh Sangwan, Li Huang, Juan Du and Liang T Chu, J Phys Chem A, 2015, 119(20), 4907-4914; DOI:10.1021/acs.jpca.5b00951 